left hemifacial numbness, and left side chewing difficulty of eight years duration. She had also progressive decline in visual acuity in the left eye for the last two years and the decline was more rapid in the last eight months. Neurological examination revealed left primary optic atrophy, left sixth nerve palsy, hyposthesia in V1, V2, and V3 distributions on the left side, and left masseter and temporalis muscle wasting and weakness.

Magnetic resonance imaging (MRI) revealed a mass lesion in the left middle cranial fossa which was of mixed intensity on $\mathrm{T} 1 \mathrm{~W}$ and $\mathrm{T} 2 \mathrm{~W}$ sequences with heterogenous contrast enhancement [Figure 1]. The mass was extending medially up to the left cavernous sinus and anteriorly up to the superior orbital fissure. Posteriorly, the lesion was extending up to the left petrous apex with compression of the left cerebral peduncle posteromedially and superiorly the lesion displaced left temporal lobe upwards with effacement of left temporal horn. MR angiogram showed complete block of the left internal carotid artery.

The patient was operated through left subtemporal intradural approach. The tumor was yellowish-white, lobulated, vascular, and moderately firm. There was a relatively good plane of cleavage. The tumor was attached at places to the dura tightly and there was erosion of temporal base. Part of the tumor was going into the infratemporal fossa. Total excision of the tumor including the involved dura was achieved and duraplasty was done [Figure 2]. The postoperative course of the patient was uneventful. At six months follow-up her headache and vision improved $(2 / 60)$ but there was no change in the other deficits.

Biopsy of the tissue revealed sparsely cellular areas of undulating spindle-shaped tumor cells with oval, mildly anisomorphic nuclei and course chromatin. The tumor cells were disposed in poorly formed vesicles on a collagenous background with small vascular channels

\title{
Giant solitary fibrous tumor of the middle cranial fossa
}

Sir,

A 33-year-old lady presented with progressive diplopia,

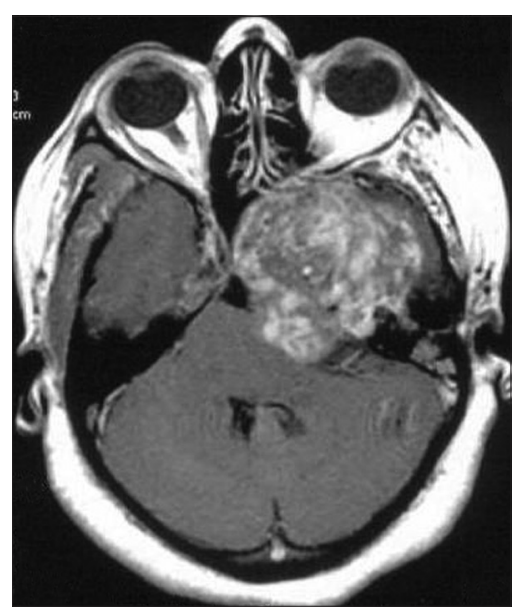

Figure 1: Contrast axial magnetic resonance imaging of the brain showing heterogenously enhancing mass in the left 


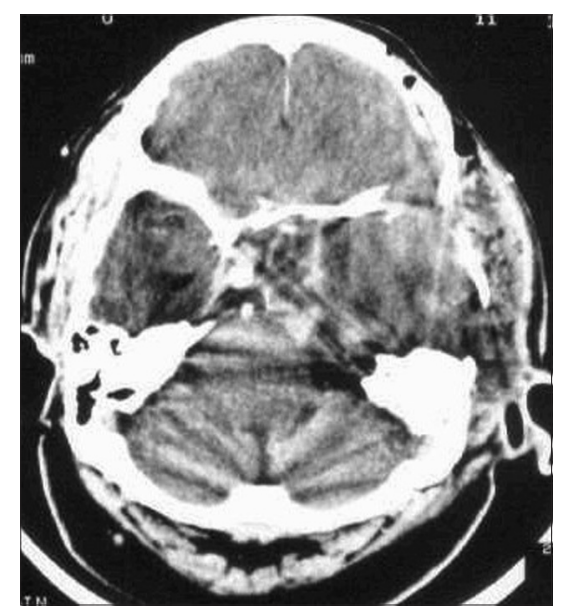

Figure 2: Postoperative contrast computed tomography scan of the brain showing complete excision of the lesion

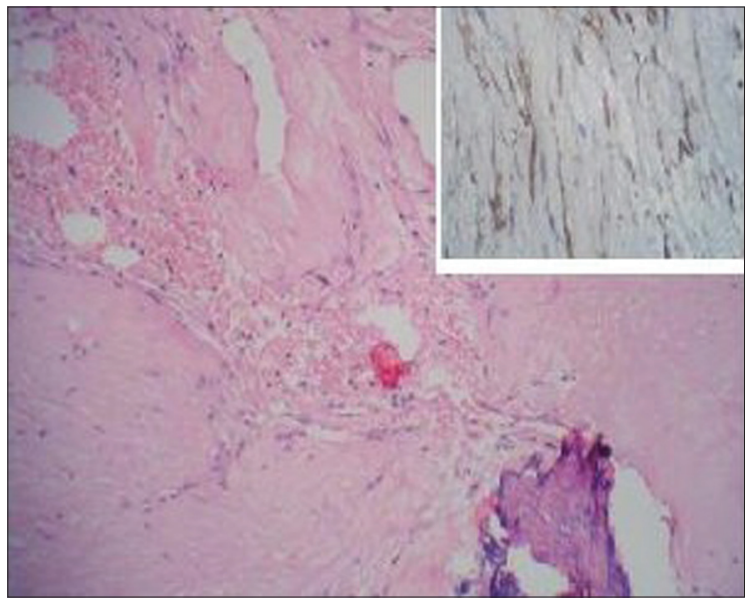

Figure 3: Photomicrograph showing fibrous elements and calcifications (H and E stain). Inset showing CD 34 immunopositivity

lined by flattened endothelial cells. There were large interspaced areas of necrosis with focal calcifications, few chronic inflammatory cells, and myxoid changes were present [Figure 3]. Immunohistochemistry was diffusely positive for CD34. Vimentin. Bcl2 immunohistochemistry was not done.

Meningeal solitary fibrous tumor (SFT) is a rare tumor and is different from fibroblastic meningiomas (FM) and hemangiopericytoma (HPC). SFTs account for $0.09 \%$ of all meningeal tumors..$^{[1-3]}$ Compared to meningiomas the SFTs show a predilection for the posterior fossa and spine. ${ }^{[1,2]}$ Most of these tumors are benign, however, local recurrence have been reported. ${ }^{[3]}$ Gengler and Guillou $^{[4]}$ in a review described SFT as one of the HPClike tumor of meninges. ${ }^{[4]}$ Differentiation of SFT from HPC is essential because of the therapeutic implications. HPC is a dural-based aggressive mesenchymal tumor and 15-year recurrence rate is $76 \%{ }^{[5]}$ Postoperative radiotherapy therefore is indicated in patients with $\mathrm{HCP}$, whereas the SFTs present a benign clinical course similar to meningiomas and do not recur unless subtotally resected. ${ }^{[2,3]}$ We reported this case of giant SFT of middle cranial fossa because of many unusual features: Rare location, local aggression, and bony erosion. The other unusual feature was internal carotid artery block. Although aggressive looking on imaging, it could be totally excised.

Rabi Narayan Sahu, A. K. Jaiswal, M. S. Sharma', S. Behari, V. K. Jain

Department of Neurosurgery, Sanjay Gandhi Post Graduate Institute of Medical Sciences, Lucknow, ${ }^{1}$ Department of Neurosurgery, All India Institute of Medical Sciences, New Delhi, India. E-mail: drrnsahu@gmail.com

\section{References}

DOI: $10.4103 / 0028-3886.59496$

1. Prayson RA, McMahon JT, Barnett GH. Solitary fibrous tumor of the meninges. Case report and review of the literature. J Neurosurg 1997;86:1049-52.

2. Brunori A, Cerasoli S, Donati R, Giangaspero F, Chiappetta F. Solitary fibrous tumor of the meninges: Two new cases and review of the literature. Surg Neurol 1999;51:636-40.

3. Carneiro SS, Scheithauer BW, Nascimento AG, Hirose T, Davis DH. Solitary fibrous tumor of the meninges: A lesion distinct from fibrous meningioma. A clinicopathologic and immunohistochemical study. Am J Clin Pathol 1996;106:217-24.

4. Gengler C, Guillou L. Solitary fibrous tumour and haemangiopericytoma: Evolution of a concept. Histopathology 2006;48:63-74.

5. Guthrie BL, Ebersold MJ, Scheithauer BW, Shaw EG. Meningeal hemangiopericytoma: Histopathological features, treatment, and longterm follow-up of 44 cases. Neurosurgery 1989;25:514-22.

Accepted on 24-03-2009 